\title{
Spatial and temporal characterization of polarization entanglement
}

DOI:

10.1142/S0219749919410272

\section{Document Version}

Accepted author manuscript

Link to publication record in Manchester Research Explorer

\section{Citation for published version (APA):}

Nomerotski, A., Katramatos, D., Stankus, P., Svihra, P., Cui, G., Gera, S., Flament, M., \& Figueroa, E. (2020). Spatial and temporal characterization of polarization entanglement. International Journal of Quantum Information, 18(1), [1941027]. https://doi.org/10.1142/S0219749919410272

\section{Published in:}

International Journal of Quantum Information

\section{Citing this paper}

Please note that where the full-text provided on Manchester Research Explorer is the Author Accepted Manuscript or Proof version this may differ from the final Published version. If citing, it is advised that you check and use the publisher's definitive version.

\section{General rights}

Copyright and moral rights for the publications made accessible in the Research Explorer are retained by the authors and/or other copyright owners and it is a condition of accessing publications that users recognise and abide by the legal requirements associated with these rights.

\section{Takedown policy}

If you believe that this document breaches copyright please refer to the University of Manchester's Takedown Procedures [http://man.ac.uk/04Y6Bo] or contact uml.scholarlycommunications@manchester.ac.uk providing relevant details, so we can investigate your claim.

\section{OPEN ACCESS}


International Journal of Quantum Information

(C) World Scientific Publishing Company

\title{
Spatial and Temporal Characterization of Polarization Entanglement
}

\author{
Andrei Nomerotski, Dimitrios Katramatos, Paul Stankus \\ Brookhaven National Laboratory, Upton NY 11973 USA anomerotski@bnl.gov \\ Peter Svihra \\ Department of Physics, Faculty of Nuclear Sciences and Physical Engineering, Czech Technical \\ University, Prague 115 19, CZ \\ Department of Physics and Astronomy, School of Natural Sciences, University of Manchester, \\ Manchester M13 9PL, UK \\ Guodong Cui, Sonali Gera, Mael Flament, Eden Figueroa \\ Department of Physics and Astronomy, Stony Brook University, Stony Brook NY 11794, USA
}

Received Day Month Year

Revised Day Month Year

\begin{abstract}
We describe the full temporal and spatial characterization of polarization-entangled photons produced by Spontaneous Parametric Down Conversions using an intensified highspeed optical camera, Tpx3Cam. This novel technique allows for precise determination of Bell inequality parameters and for new characterization methods for the spatial distribution of entangled quantum information. We also discuss a technique to synchronize multiple cameras separated by vast distances, which will be required for a distributed quantum network.
\end{abstract}

Keywords: Tpx3Cam; entanglement.

\section{Introduction}

Quantum networking, one of four pillars of Quantum Information Science along with quantum computing, quantum sensors, and quantum algorithms, has seen a great deal of development over the past several years, both in its goals and its techniques ${ }^{[1]}$ Most generally, quantum networking is the ability to take two (or more) physical qubits in an entangled, e.g. non-factorizable, state and reliably transport them across large macroscopic distances ( meters to multi-kilometers) while preserving the multi-particle quantum state. In the experiments described here the macroscopic quantum state is a two-photon pair produced through a standard spontaneous parametric down-conversion with their polarizations entangled at creation, and then transported over meter-length fiber optics. The preservation of two-particle entanglement after transport is demonstrated via a standard analysis with adjustable polarizers to show violation of a Bell inequality 4 (5) (specifically 
the Clauser-Horne-Shimony-Holt (CSHS) inequality, see below) and thus the persistence of non-classical long-distance correlations.

Two-mode entanglement can be extended to more complex situations, involving multiple modes in spatial, temporal and polarization degrees of freedom $[6] 10] \mathrm{In}$ our study we also investigate, beyond the two-channel analysis, the entanglement correlation signature between photons detected at different spatial locations within the two beams after being released from their optical fiber channels $11-15$ This is accomplished using a "fast camera", single-photon-sensitive pixel detector ${ }^{16}$ with nano-second scale arrival time resolution to separate detections within a single camera sensor. The result is a simultaneous spatial and temporal coincidence measurement of polarization entanglement between a pair of macroscopically separated photons.

\section{Spatially resolved Bell State Measurement}

We discuss here the experimental technique used and the results obtained, with topics in the following order:

- Experimental setup; description of the entangled pair source and the fast pixel camera detector (Sec. 2.1)

- Basic demonstration of entanglement via polarization analysis for the CSHS inequality, using one camera for both detections (Sec. 2.2)

- Measurement of pair entanglement between pairs at different spatial locations in pixel detector (Sec. 2.3.

The extension of the measurement to use two pixel cameras at separate locations, including techniques for remote synchronization, are then discussed further in Section 3.

\subsection{Experimental Setup}

The SPDC source (QuTools QuED) utilizes a blue pump laser diode tuned to the wavelength of $405 \mathrm{~nm}$, and a pair of Type I non-collinear BBO crystals with optical axes perpendicular to one another to generate signal and idler photons entangled in polarization at a wavelength of $810 \mathrm{~nm}$. The first crystal optical axis and the pump beam define the vertical plane, so an incoming photon which is vertically polarized gets down-converted and produces two horizontally polarized photons in the first crystal. In contrast, a horizontally polarized photon produces two vertically polarized photons. Signal and idler photons are spatially separated and collected using single-mode fibers, with a linear polarizer used for projective measurements. The rate of entangled photon pairs from the source is about $10 \mathrm{kHz}$.

Registration of single photons and characterization of the sources in the experiments was performed using an intensified time stamping camera with single photon sensitivity, Tpx3Cam $\sqrt{17}$ The camera is a hybrid pixel detector: an optical sensor 
with high quantum efficiency ${ }^{19}$ is bump-bonded to a Timepix3 ASIC, 20 a timestamping readout chip with $256 \times 25655 \times 55 \mu \mathrm{m}^{2}$ pixels. The processing electronics in each pixel records the ToA (time of arrival) of hits that cross a preset threshold with $1.6 \mathrm{~ns}$ resolution and stores it as a timecode in a memory inside the pixel. The information about ToT (time-over-threshold), deposited energy in each pixel, is also stored. The readout is data driven with only $T=475 \mathrm{~ns}+$ TOT pixel dead time, which allows multi-hit functionality at the pixel level and fast, $80 \mathrm{Mpix} / \mathrm{sec}$, throughput. Tpx3Cam can also accept and time stamp an external trigger pulse, independent of the Timepix 3 pixels. The granularity of the trigger time measurement is $0.26 \mathrm{~ns}$.

The camera was calibrated to equalize the response of all pixels by adjusting the individual pixel thresholds. After this procedure, the effective threshold to fast light flashes from the intensifier is $600-800$ photons per pixel depending on the wavelength. A small $(\approx 0.1 \%)$ number of hot pixels was masked to prevent logging large rates of meaningless data.

In the single photon sensitive operation, the camera is coupled to a cricket with an intensifier and relay optics to project the light flashes from the intensifier output screen directly on to the optical sensor in the camera. The image intensifier is a vacuum device with a photocathode followed with micro-channel plate (MCP) and fast scintillator, which ensures single photon sensitivity. The quantum efficiency (QE) of the intensifier hi-QE-red photocathode (Photonis) is $18 \%$ at $800 \mathrm{~nm}$. The MCP efficiency is close to $100 \%$ and light flashes produced by each photon in the intensifier have plenty of signal to cross the Timepix 3 threshold so the overall detection efficiency of single photons in the setup should be mostly determined by the photocathode QE. Similar configurations of the intensified Tpx3Cam was before used for the quantum illumination ${ }^{21}$ and lifetime imaging ${ }^{221}$ studies.

\subsection{Characterization of polarization entanglement}

We used the camera to spatially characterize photonic polarization entanglement of the SPDC photon source mentioned above ${ }^{16}$ The two beams of photons from the fibers were detected by the same camera. Figure 1 shows a photograph of Tpx3Cam with two fibers pointing to the photocathode. The graph on the right in the same figure shows the occupancy map of the sensor $(256 \times 256$ pixels $)$ for the full statistics of a five-minute run, the color encodes the number of times a particular pixel was hit in decimal log scale. The two beam spots coming from the optical fibers are clearly visible, corresponding to the areas of highest occupancy. The graph also shows how the round $18 \mathrm{~mm}$ diameter output window of the intensifier is mapped onto the $14 \mathrm{~mm} \times 14 \mathrm{~mm}$ optical sensor in the camera. This is visible due to the intensifier dark count rate, about $20 \mathrm{kHz}$ over the full area at room temperature for the used photocathode.

To identify pairs of simultaneous photons we selected areas of the sensor cor- 

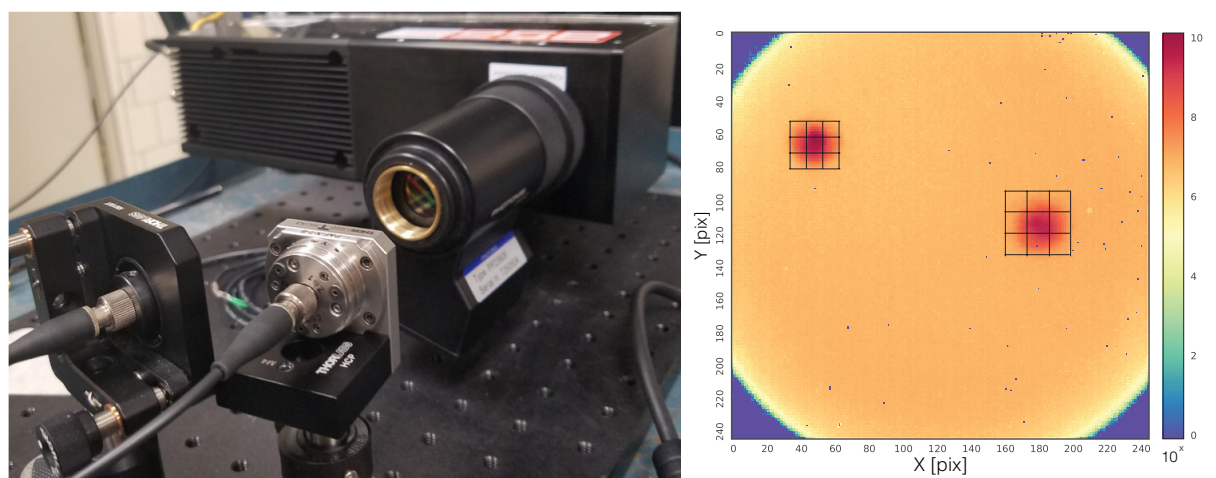

Fig. 1. Left: Photograph of Tpx3Cam with two fibers pointing to the intensifier photocathode. Right: Occupancy map of the sensor $(256 \times 256$ pixels $)$ showing the photon hits for the full statistics of a five-minute run, the color encodes the number of times a particular pixel was hit in decimal $\log$ scale.

responding to regions illuminated by the fibers. Then, for each photon detected in one region, we looked for its associated pair at the closest time in the second region. This procedure as well as other data processing steps are discussed in detail elsewhere $\frac{16}{16}$ The distribution of the time difference of the two photons has a peak at 0 corresponding to the pairs of entangled photons as shown in the left graph of Figure 2 for several combinations of photon polarization. Each distribution was fit to a function consisting of two Gaussians and a constant accounting for the flat background of random coincidences.

The next step consists in studying the dependence of the coincidence measurements on the polarization projective measurements of the two-photon state. In the measurements, one polarizer was varied in $20^{\circ}$ steps for four fixed values of the other polarizer: $0^{\circ}, 45^{\circ}, 90^{\circ}$ and $135^{\circ}$. The dependence of the number of coincidences versus the polarizer angle is shown in the right graph of Figure 2. The data points were fitted with a sine function with the period, phase, amplitude and offset as free parameters, which gives the dependence of the coincidence signal on polarization projective measurements of the two photon state. We note that there is a difference in amplitudes for different cases since the produced state is not a pure $(H H+V V) / \sqrt{2}$ state but has a small admixture of $(H H-V V) / \sqrt{2}$ due to non-ideal experimental conditions.

Our next step is to calculate the Clauser-Horne-Shimony-Holt (CHSH) inequality violation $23 \sqrt[24]{24}$ using the above results. The inequality can be written as:

$$
S=E(\alpha, \beta)+E\left(\alpha^{\prime}, \beta\right)-E\left(\alpha, \beta^{\prime}\right)+E\left(\alpha^{\prime}, \beta^{\prime}\right) \leq 2
$$

where

$$
E(\alpha, \beta)=\frac{N_{V V}(\alpha, \beta)+N_{H H}(\alpha, \beta)-N_{V H}(\alpha, \beta)-N_{H V}(\alpha, \beta)}{N_{V V}(\alpha, \beta)+N_{H H}(\alpha, \beta)+N_{V H}(\alpha, \beta)+N_{H V}(\alpha, \beta)}
$$



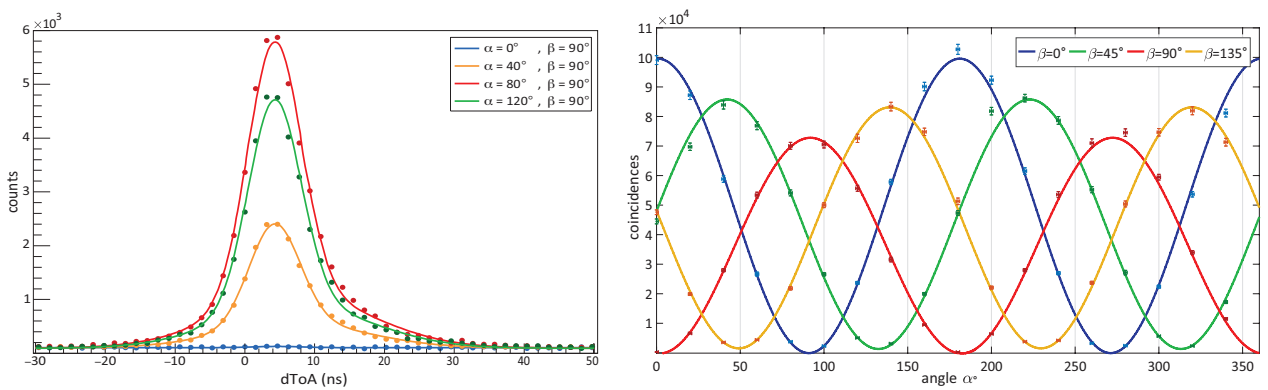

Fig. 2. Left: Distribution of time difference between two photons in different fibers for selected pairs of polarization settings combinations: $\alpha=0^{\circ}$ (blue), $\alpha=40^{\circ}$ (orange), $\alpha=80^{\circ}$ (red) and $\alpha=120^{\circ}$ (green) with $\beta=90^{\circ}$. The amplitudes for different polarization combinations are determined by the entangled state projection. Flat background results from uncorrelated photon background. The total number of coincidences is calculated by integration over the Gaussian curves. Right: Coincidences as a function of polarization: showing the dependence of the signal amplitude (number of coincidences) for different settings of a Clauser-Horne-Shimony-Holt type Bell inequality test.

from the fitted curves in Figure $2 . N_{x y}(\alpha, \beta)$ with $x, y=V, H$ are defined to represent counting schemes for linear polarizer angles such that $N_{V V}(\alpha, \beta)=N(\alpha, \beta)$, $N_{H V}(\alpha, \beta)=N\left(\alpha+90^{\circ}, \beta\right), N_{V H}(\alpha, \beta)=N\left(\alpha, \beta+90^{\circ}\right)$, and $N_{H H}(\alpha, \beta)=$ $N\left(\alpha+90^{\circ}, \beta+90^{\circ}\right)$.

The obtained value is $2.78 \pm 0.02$, well above the classical limit of 2 and close to the Tsirelson Bound of $2 \sqrt{2}(2.82)$. We note that the applied analysis technique allowed for the better rejection of random background compared to more traditional analysis. In these measurements, the signal is estimated with a fitting procedure described in the previous section. This procedure automatically accounts for the background and the camera time resolution using the same dataset and gives an unbiased estimate of the signal, which is not the case for the standard analysis.

\subsection{Spatial sub-detection}

One of the clear advantages of using high-speed cameras for quantum state characterization lies in the capacity to analyze multiple processes simultaneously. In our next experiment, we probed the ability of the fast camera to analyze 81 entangled pairs in parallel. To simulate the latter, we divided the areas illuminated by the fiber's output into nine subareas, forming a $3 \times 3$ matrix as shown in the two fiber regions in the right graph of Figure 1 . Then, we analyzed each pair-wise combination (81 total combinations) independently and reproduced the Bell analysis presented above for each of them. To accumulate enough statistics for these spatially resolved measurements, we took one-hour-long extended datasets, corresponding to the 16 combinations of the polarizers settings, which are needed to calculate the Bell's inequality violation. Figure 3 shows the results of the parallel evaluation of 81 Bell's inequalities. 


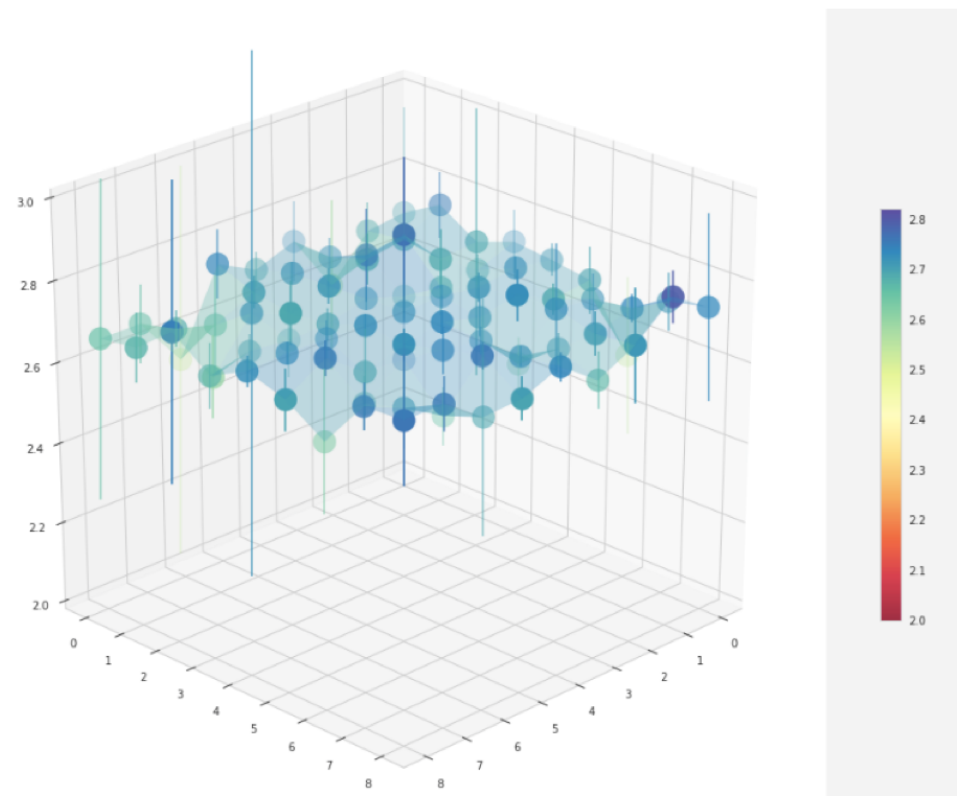

Fig. 3. Spatial characterization of S-values as measured by Tpx3Cam. In this configuration the two areas on the fast camera that are illuminated by photons from the fibers are divided into subareas, forming two $3 \times 3$ matrices. The coincidence thus decomposes into that of 81 possible pairs of a combination of subareas. Using these coincidences, we calculated the CHSH inequality violation and plotted the resulting $S$-values in the form of 81 blocks in nine $3 \times 3$ matrices.

Each point in the graph represents a specific spatial combination of subareas. The corresponding S-value is color-coded with the corresponding uncertainty shown in the center of the box. The results show that the S-value is uniform within the experimental errors with no apparent position dependence as expected. We also checked that the values of $E(\alpha, \beta)$ correlators from Eq.(2) do not have position dependence either. Thus, since the camera can provide simultaneously spatially and temporarily resolved photon pairs we were able to perform a multi-dimensional measurement of the S-value in a single dataset.

\section{Measurements with two fast cameras}

Considerable distances could separate the quantum devices, so characterization techniques that rely on multiple single-photon detectors are essential. For the timeresolved measurements, those detectors must be synchronized with respect to each other with reasonable accuracy.

For the fast cameras such as used in the above measurements, the provided time stamps rely on the internal oscillator with typical stability of a few ppm (part per million). This corresponds to a few microsecond relative drift of time references for two cameras only after a second of operation and, therefore, is not acceptable since 

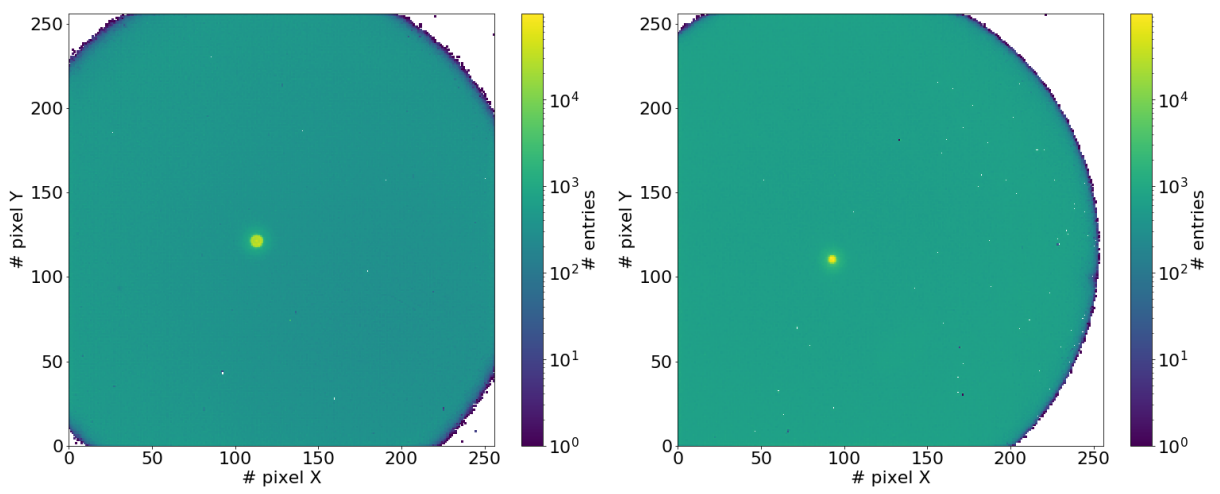

Fig. 4. Signal from the fibers in two Tpx3Cam cameras.

typical measurements can last hours and require nanosecond accuracy.

To solve this problem we investigated ways to synchronize the cameras. The test system employed the same SPDC source as before connected to two few hundred meter long fibers. The photons pairs after the fibers were registered using two independent Tpx3Cam cameras. Figure 4 shows the occupancy plots from two Tpx3Cam cameras with the photon signals clearly visible in the center. The first camera employed the same hi-QE-Red intensifier as described above while the second camera had a similar intensifier but with the S25 photocathode (Photonis), which had a smaller QE of about $8 \%$ at $810 \mathrm{~nm}$.

We used the White Rabbit (WR) system ${ }^{25}$ developed at CERN for the synchronization of the cameras. The WR modules are placed in the same locations as the cameras and are connected to each other via a separate optical fiber. After a calibration the modules start generating a $1 \mathrm{~Hz}$ pulses (PPS) and $10 \mathrm{MHz}$ clock, which can be used as absolute time reference and provide sub-ns precision of synchronization. The PPS signal was used for the camera's trigger inputs to be time-stamped with 0.26 ns precision synchronously with the pixel time-stamping. This "trigger" information is inserted into the recorded datasteam together the pixel dat ${ }^{26}$ so can be conveniently used for synchronization.

The data analysis followed the same steps as described in the previous section but in this case the fiber regions were selected in the two different cameras. Figure 5 shows the time difference between two photons from both regions of interest in the cameras. The bottom left graph shows the scatter plot of the time difference between photon pairs as function of ToF (time of flight after the 1 pps synchronization pulse) before applying any correction and the top left graph is the corresponding projection on the time difference axis.

One can see that the temporal drift is considerable, of the order of 1 microsecond over the duration of one second. However, the dependence has a constant slope so it can be fitted with a linear function providing an efficient correction of this temporal drift of synchronization. The graphs on the right show the same information after 

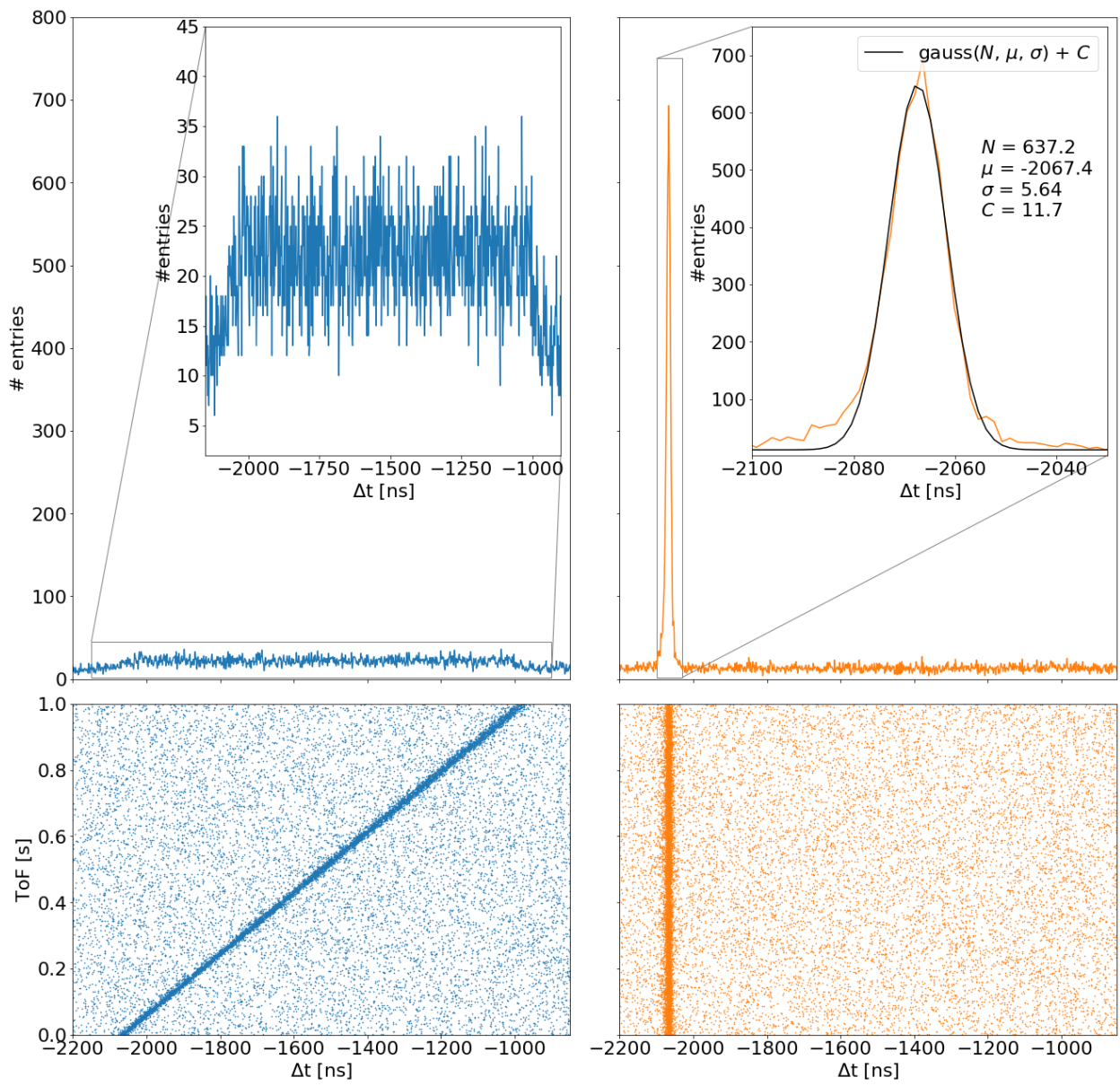

Fig. 5. Effect of the relative clock drift on the coincidence timing resolution. The bottom left plot shows the scatter plot of the coincidences time difference $\Delta t$ as a function of time relative to the White Rabbit synchronization $1 \mathrm{~Hz}$ pulses. One can see that the relative time drift is considerable $-\mathcal{O}(1 \mu \mathrm{s})$ over the duration of $1 \mathrm{~s}$. Since even over long duration of data-taking $\mathcal{O}(100 \mathrm{~s})$, the slope of the drift is constant and can be efficiently corrected with a linear function. The bottom right graphs show the same information after the correction is applied, top plots show projection of $\Delta t$. The insert in the top right graph shows details of a Gaussian fit to the coincidence peak.

the linear correction is applied, and the improvement is remarkable indeed.

Length of the fibers connecting the cameras to the SPDC source were not equal. This led to a non-zero time difference for the coincidences of 2067 nanosecond as shown in the insert in Figure 5 . The linear correction accounting for the relative time drift of two cameras recovers the time resolution. The insert in Figure 5 shows the time difference between two photons from the source after the drift and TOT corrections. The TOT correction is needed to account for a more subtle dependence of the measured TOA in the pixel on the signal magnitude in this pixel, and it is 
described in detail elsewhere $\frac{18}{18}$

The coincidence peak and flat background are fit in the same manner as in the previous section with a Gaussian to account for the coincidence signal and a constant to account for the flat background of accidentals. The time resolution (sigma of the Gaussian in the fit) was determined to be equal to $5.6 \mathrm{~ns}$, a factor of two worse than in the case of shorter fibers $\frac{16}{16}$ most likely due to the residual non-linearity of the clock drift correction. Work is in progress to improve it.

\section{Summary}

We have demonstrated a novel technique that provides spatially and temporally resolved information for characterization of photonic polarization entanglement employing the intensified Tpx3Cam camera. The camera can simultaneously timestamp multiple single optical photons with $\mathrm{MHz}$ throughput and nanosecond timing resolution while capturing their spatial information. Several cameras can be efficiently synchronized while preserving their excellent time resolution to single photons. The S-value results confirm that the fast camera spatial characterization of quantum-states in parallel is a viable alternative to be used in scaled-up quantum systems.

\section{Acknowledgments}

The authors thank A. Hardy, A. Londono, J. Tsybysheva and Y. Kim for their assistance with the measurements; H. Graafsma for providing the Timepix3 ASIC; M. van Beuzekom, B. Bouwens, and E. Maddox for their assistance with the fast camera; T. Tsang for assistance with the image intensifiers. The Stony Brook team acknowledges the support from the National Science Foundation (grants PHY1404398 \& PHY-1707919) and the Simons Foundation (grant SBF-241180). The Czech Ministry of Education also supported this work, Youth and Sports (grant LM2015054) and by the LDRD grant 18-051 of Brookhaven National Laboratory.

\section{References}

1. W. Tittel, J. Brendel, H. Zbinden, and N. Gisin. Violation of bell inequalities by photons more than $10 \mathrm{~km}$ apart. Phys. Rev. Lett., 81:3563-3566, Oct 1998.

2. Jian-Wei Pan, Dik Bouwmeester, Harald Weinfurter, and Anton Zeilinger. Experimental entanglement swapping: Entangling photons that never interacted. Phys. Rev. Lett., 80:3891-3894, May 1998.

3. T. Ndousse-Fetter, N. Peters, et al. Quantum networks for open science. arXiv:1910.11658 [quant-ph], 2019.

4. R. Ursin, F. Tiefenbacher, T. Schmitt-Manderbach, H. Weier, T. Scheidl, M. Lindenthal, B. Blauensteiner, T. Jennewein, J. Perdigues, P. Trojek, B. Omer, M. Furst, M. Meyenburg, J. Rarity, Z. Sodnik, C. Barbieri, H. Weinfurter, and A. Zeilinger. Entanglement-based quantum communication over 144km. Nat Phys, 3(7):481-486, Jul 2007. 
5. B. Hensen, H. Bernien, A. E. Dréau, A. Reiserer, N. Kalb, M. S. Blok, J. Ruitenberg, R. F. L. Vermeulen, R. N. Schouten, C. Abellán, W. Amaya, V. Pruneri, M. W. Mitchell, M. Markham, D. J. Twitchen, D. Elkouss, S. Wehner, T. H. Taminiau, and R. Hanson. Loophole-free bell inequality violation using electron spins separated by 1.3 kilometres. Nature, 526:682, Oct 2015.

6. Claude Fabre and Nicolas Treps. Modes and states in quantum optics. 2019 https://arxiv.org/abs/1912.09321.

7. Salvatore Virzì, Enrico Rebufello, Alessio Avella, Fabrizio Piacentini, Marco Gramegna, Ivano Ruo Berchera, Ivo Pietro Degiovanni, and Marco Genovese. Optimal estimation of entanglement and discord in two-qubit states. Sci. Rep. 9, 3030 (2019), 9, Feb 2019.

8. Marco Genovese. Research on hidden variable theories: A review of recent progresses. Phys. Rep., 413:319-396, July 2005.

9. G. Brida, M. Genovese, L. A. Krivitsky, and M. V. Chekhova. Interference structure of two-photon amplitude revealed by dispersion spreading. Phys. Rev. A, 75:015801, Jan 2007.

10. Paul-Antoine Moreau, Ermes Toninelli, Thomas Gregory, Reuben S. Aspden, Peter A. Morris, and Miles J. Padgett. Imaging bell-type nonlocal behavior. Science Advances, $5(7), 2019$

11. Giorgio Brida, Ivo Pietro Degiovanni, Angela Florio, Marco Genovese, Paolo Giorda, Alice Meda, Matteo G. A. Paris, and Alexander Shurupov. Experimental estimation of entanglement at the quantum limit. Phys. Rev. Lett., 104:100501, Mar 2010.

12. Karol Bartkiewicz, Bohdan Horst, Karel Lemr, and Adam Miranowicz. Entanglement estimation from bell inequality violation. Phys. Rev. A, 88:052105, Nov 2013.

13. Giorgio Brida, Ivo P. Degiovanni, Angela Florio, Marco Genovese, Paolo Giorda, Alice Meda, Matteo G. A. Paris, and Alexander P. Shurupov. Optimal estimation of entanglement in optical qubit systems. Phys. Rev. A, 83:052301, May 2011.

14. G Brida, M Genovese, L A Krivitsky, M V Chekhova, and E Predazzi. Go and return propagation of biphotons in fibre and polarization entanglement. Journal of Physics A: Mathematical and Theoretical, 40(28):7985-7992, jun 2007.

15. G. Brida, M. V. Chekhova, M. Genovese, M. Gramegna, and L. A. Krivitsky. Dispersion spreading of biphotons in optical fibers and two-photon interference. Phys. Rev. Lett., 96:143601, Apr 2006.

16. C. Ianzano et al. Spatial characterization of photonic polarization entanglement using a fast camera, 2018. https://arxiv.org/pdf/arXiv:1808.06720.pdf.

17. M. Fisher-Levine and A. Nomerotski. Timepixcam: a fast optical imager with timestamping. Journal of Instrumentation, 11(03):C03016, 2016.

18. A. Zhao et al. Coincidence velocity map imaging using tpx3cam, a time stamping optical camera with 1.5 ns timing resolution. Review of Scientific Instruments, 88(9):113104, November 2017.

19. A. Nomerotski, I. Chakaberia, M. Fisher-Levine, Z. Janoska, P. Takacs, and T. Tsang. Characterization of timepixcam, a fast imager for the time-stamping of optical photons. Journal of Instrumentation, 12(01):C01017, 2017.

20. T. Poikela et al. Timepix3: a $65 \mathrm{k}$ channel hybrid pixel readout chip with simultaneous toa/tot and sparse readout. Journal of instrumentation, 9(05):C05013, 2014.

21. Y. Zhang et al. Multidimensional quantum illumination via direct measurement of spectro-temporal correlations, 2018. https://arxiv.org/pdf/1909.09664.pdf.

22. Rajannya Sen, Liisa M. Hirvonen, Alexander Zhdanov, Peter Svihra, Stefan Andersson-Engels, Andrei Nomerotski, and Dmitri Papkovsky. New luminescence lifetime macro-imager based on a tpx3cam optical camera. Biomed. Opt. Express, 
11(1):77-88, Jan 2020

23. Alain Aspect, Philippe Grangier, and Gérard Roger. Experimental tests of realistic local theories via bell's theorem. Phys. Rev. Lett., 47:460-463, Aug 1981.

24. Alain Aspect, Philippe Grangier, and Gérard Roger. Experimental realization of einstein-podolsky-rosen-bohm gedankenexperiment: A new violation of bell's inequalities. Phys. Rev. Lett., 49:91-94, Jul 1982.

25. P. Moreira, J. Serrano, T. Wlostowski, P. Loschmidt, and G. Gaderer. White rabbit: Sub-nanosecond timing distribution over ethernet. In 2009 International Symposium on Precision Clock Synchronization for Measurement, Control and Communication, pages $1-5$, Oct 2009 .

26. B. van der Heijden et al. Spidr, a general-purpose readout system for pixel asics. Journal of instrumentation, 12(02):C02040, 2017. 